\title{
Acute generalized exanthematous pustulosis
}

\author{
Kristyna Cleminson MD, Natalie Cunningham MD
}

Cite as: CMAJ 2020 October 19;192:E1268. doi: 10.1503/cmaj.200324

A $\mathrm{n}$ otherwise healthy 71-year-old woman with bradycardia was admitted to hospital for placement of a pacemaker. The patient received 1 dose of cefazolin during the procedure. The next day, a pruritic morbilliform eruption developed on her lower extremities. Two days later, the eruption became widespread with confluent erythematous plaques and overlying sheets of nonfollicular pustules (Figure 1). Her mucous membranes were not affected. She was febrile with leukocytosis (34 [normal range 4.5-11] $\times 10^{9} / \mathrm{L}$ ). We diagnosed acute generalized exanthematous pustulosis related to cefazolin, and this was supported by histologic examination of the tissue, which showed subcorneal pustules, spongiosis, perivascular neutrophils and eosinophilic exocytosis. ${ }^{1}$ Our patient did not receive any further doses of cefazolin, and we prescribed betamethasone cream $(0.1 \%)$ to be applied to the rash twice daily for 2 weeks. Systemic steroids were not used. Her rash resolved completely.

Acute generalized exanthematous pustulosis is an uncommon, severe type IV hypersensitivity drug reaction associated with antibiotics, calcium channel blockers, antimalarials, acetaminophen, nonsteroidal anti-inflammatory drugs and terbinafine. It starts abruptly, within 2-5 days of drug exposure. The rash is characterized by nonfollicular pustules on an erythematous base, usually involving intertriginous areas. ${ }^{2}$ Pustules last a few days before superficial desquamation of all involved areas; mucous membranes are usually spared. ${ }^{3}$ Patients often describe itchiness and burning. ${ }^{1,3}$ Renal and hepatic involvement has been described. ${ }^{1,2}$ The differential diagnosis includes infection, pustular psoriasis and toxic epidermal necrolysis. ${ }^{1,3}$

The condition usually resolves within 2 weeks; however, among frail patients who develop fever and superinfection, it has a mortality rate of $5 \% .^{2}$ After recovery, patch testing is $80 \%$ sensitive and $85 \%$ specific in identifying causative agents. ${ }^{1,2}$ Treatment includes stopping the offending drug, antipyretics and topical or systemic corticosteroids to decrease pruritus and inflammation. . $^{2,3}$

\section{References}

1. Speeckaert MM, Speeckaert R, Lambert J, et al. Acute generalized exanthematous pustulosis: an overview of the clinical, immunological and diagnostic concepts. Eur J Dermatol 2010;20:425-33.

2. De A, Das S, Sarda A, et al. Acute generalized exanthematous pustulosis: an update. Indian J Dermatol 2018;63:22-9.

3. Sidoroff A, Halevy S, Bavinck J, et al. Acute generalized exanthematous pustolusis (AGEP) - a clinical reaction pattern. J Cutan Pathol 2020;28:113-9.

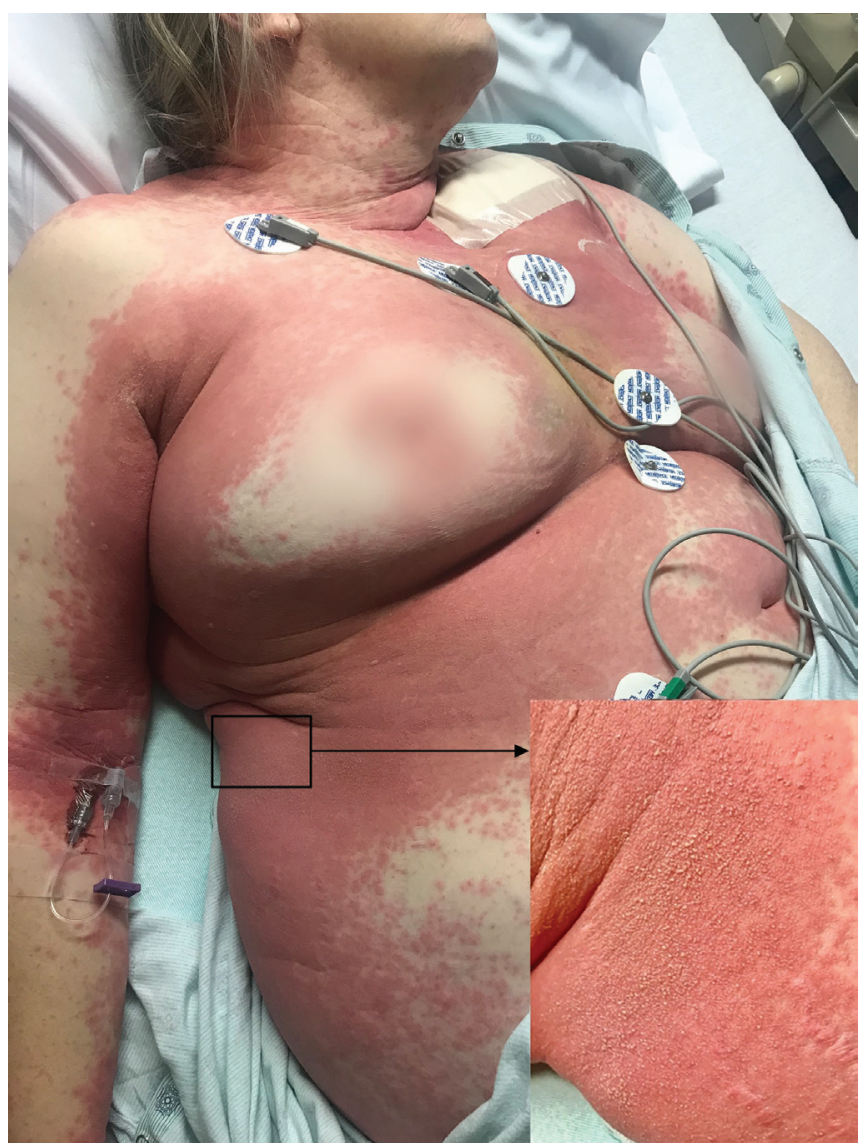

Figure 1: Widespread erythematous eruption, favouring the flexural surfaces, with overlying sheets of nonfollicular pustules in a 71-year-old woman with acute generalized exanthematous pustulosis.

\section{Competing interests: None declared.}

This article has been peer reviewed.

The authors have obtained patient consent.

Affiliation: Division of Clinical Dermatology \& Cutaneous Science, Department of Medicine, Dalhousie University, Halifax, NS

Correspondence to: Kristyna Cleminson, k.cleminson@dal.ca 